\title{
INTEGRACIÓN EUROPEA Y PROGRESO EN CONSTRUCCIÓN ARQUITECTÓNICA (A propósito de un tema pendiente: la adecuación acústica de nuestros edificios)
}

\author{
(ON EUROPEAN INTEGRATION AND PROGRESS IN ARCHITECTURAL \\ CONSTRUCTION)
}

José Luis González Moreno-Navarro, Dr. Arquitecto

Prof. Titular de Construcción Arquitectónica de la Universidad Politécnica de Catalunya. Barcelona/España.

\section{RESUMEN}

Conseguir que la incorporación del sector de la edificación al Mercado único se realice de forma amplia y efectiva, requerirá no sólo una renovación de nuestra realidad tecnológica y normativa, sino también un notable cambio de muchos de los valores y pautas mentales de todos los que intervienen en el diseño arquitectónico y en los procesos de construcción y contratación. Un ámbito en el que todo ello se pone muy de manifiesto es el de la protección del ruido, uno de los seis requisitos esenciales según la Directiva europea, regulado aquí por una norma no sólo alejada de las europeas, sino totalmente inoperante, y poco o nada considerada por la mentalidad casi exclusivamente figurativa de los arquitectos.

Estas han sido algunas de las ideas dominantes durante el presente curso de la asignatura Construcción IV (tardes) de la ETSAB.

\section{SUMMARY}

The full and effective incorporation of the building sector to the Single Market, will require not only a technological and normative renewal but also a significant change in the values and mental patterna of all those who participate in architectural design and in the construction and contracting processes. An area where this becomes very apparent is in the protection against nolse, one of the six essential requirements according to the European Directive, regulated here by a norm that is not only removed from the European ones, but also completely inoperative, and considered little or not all by the almost exclusively figurative mentality of architects.

These were some of the dominating ideas during the present course of the subject CONSTRUCTION IV (afternoons) of the ETSAB.

\section{INTEGRACIÓN Y COLONIZACIÓN ENCUBIERTA}

La incorporación al Mercado único europeo habrá de suponer, en el ámbito de la construcción arquitectónica, un cambio notable de las pautas mentales que rigen las decisiones de carácter formal, tecnológico y contractual.

Sin embargo, y en contraste con la gran cantidad de literatura que se está generando en relación a ello en otros países europeos, aquí no parece que sea un te$\mathrm{ma}$ al que se le dedique una atención proporcionada a su trascendencia 1 .

Este artículo parte de considerar que ha de ser un tema prioritario, si no queremos que la incorporación comporte en determinados ámbitos una colonización técnico-cultural encubierta. Una colonización que no sólo podrá afectar a los productos manufacturados, sino también a aspectos más sutiles, tales como los modelos de división del conocimiento tecnológico.

Aunque es probable que la estructura profesional tienda a unificarse, es difícil pensar que lo haga en pocos lustros. Las diferencias entre las maneras alemana, inglesa o francesa de descomponer profesionalmente el conocimiento, y la española, basada en un arquitecto de amplio espectro, será un factor clave más a considerar, según los casos, en contra o en favor nuestro.

$Y$ donde todo ello puede tener mayor trascendencia es en los ámbitos de la construcción arquitectónica cuyo conocimiento científico y tecnológico ha quedado sin apenas desarrollo entre nosotros. La adopción de pa- 
rámetros y soluciones europeas no adaptadas a nuestro entorno será el efecto no deseado pero irremediable. Y, al contrario, Niveles europeos sí deseables no podrán alcanzarse si no conseguimos un mayor conocimiento de nuestras condiciones, tanto culturales como tecnológicas, junto con la oportuna reconversión de éstas.

Uno de esos ámbitos olvidados es el que abarca todo lo relacionado con la habitabilidad y su control mediante cerramientos. Dejando para otra ocasión el profundizar en las causas, baste ahora decir que es una lógica consecuencia de la prioridad absoluta, en cuanto a investigación, que han disfrutado en nuestro país las cuestiones relacionadas con la seguridad y eficacia de las estructuras, especialmente de hormigón.

$\mathrm{Y}$, sin duda, dentro del ámbito de la habitabilidad, donde todo queda más patente es en lo relacionado con la protección del ruido, requisito prioritario desde hace décadas en muchos países europeos, y requisito esencial ahora para la integración europea, pero especialmente olvidado por nuestra realidad investigadora ${ }^{2}$.

Las consecuencias de todo ello pueden ser variadas como, por ejemplo, que en los próximos años se produzca una invasión de componentes aislantes de fachadas que ignoren nuestras necesidades estivales, o como que constatemos finalmente que con nuestras maneras constructivas es imposible alcanzar la intimidad acústica que pueden disfrutar los alemanes.

\section{LA DIFICIL UNIFICACIÓN DE LA VARIABLE CULTURAL}

Como es bien sabido, la necesaria libertad de circulación comercial de los productos y materiales de construcción, queda inicialmente regulada en sus aspectos más abstractos y generales, por la Directiva del 21 de diciembre de 1988, y complementada por los Documentos Interpretativos particulares, cuya publicación definitiva se espera para finales de este año 1991.

La esencia del cambio que implica todo ello en el ámbito normativo, se puede enunciar diciendo que, de normalizar el cómo se construye se pasa a normalizar en abstracto el objetivo que se debe alcanzar con la construcción, es decir, el qué se debe conseguir.

La principal dificultad planteada es la unificación de los criterios que han de permitir la valoración de los productos de construcción, una vez colocados en su lugar definitivo, según contribuyan u obstaculicen la consecución de los objetivos particulares y generales. $Y$ es de tal magnitud que no falta quien opina que realmente será imposible.
No obstante la dificultad es variable según el requisito esencial de que se trate. En algunos de ellos ya se ha avanzado suficientemente como para que se prevea un fácil progreso hasta la integración total, que a la larga no suponga ningún cambio en las pautas mentales vigentes actualmente. En el caso del requisito de la Resistencia mecánica y Estabilidad con un cuerpo unificador muy adelantado, ya que, además de existir desde hace décadas organismos supranacionales realmente efectivos, el objetivo a cumplir es siempre el mismo en cualquiera de las zonas geográficas, con la única variación de la sismicidad.

Sin embargo, otros en los que las diferencias climáticas y culturales son variables decisivas, todavia están en sus más difíciles inicios. Tal es el caso del ámbito de la durabilidad, en el que es realmente difícil introducir los mecanismos que valoren la adecuación de un producto según el clima y el microclima en el que está inmerso o según las prácticas habituales en el lugar en relación al mantenimiento ${ }^{3}$.

Pero sin duda, los ámbitos en los que la variable dominante es la idea de confort o comodidad son los que plantean mayores dificultades, y más todavía si van asociados a la variable climática. Tal es el caso del ámbito del confort térmico, o especialmente el del confort acústico al que, además de sus propias características fuertemente subjetivas, se le suman, en las regiones más cálidas, las del térmico.

Si bien la integración en el Mercado único no ha de implicar la unificación de los niveles de exigencia, sí parece lógico que la mayor proximidad tecnológica consecuente, y la unificación de los procedimientos de medición ${ }^{4}$, incentivará al menos una aproximación.

Y es en los ámbitos de la habitabilidad donde mayor debe ser la creatividad de los que asumen en nuestro país la responsabilidad de la creación formal y tecnológica, con tal de que esa aproximación cuente con nuestra realidad y no suponga una imposición de criterios ajenos. Pero eso es precisamente lo que no ocurre especialmente en la consecución de la placidez acústica.

\section{LA PLACIDEZ ACÚSTICA, ASIGNATURA PENDIENTE}

La incidencia de factores de economía ha favorecido que las exigencias térmicas hayan sido más consideradas tanto por creadores formales como por tecnólogos y normalizadores, pese a su flagrante olvido en los presupuestos iniciales del Movimiento Moderno y consecuente falta de valor en la modernidad ${ }^{5}$. A pesar de todo, en la segunda mitad del siglo se ha generado una manera de diseñar, por supuesto no seguida por mu- 
chos figurativos, que conecta a su vez con las formas arquitectónicas y constructivas tradicionales decantadas durante siglos también por la adecuación climática.

Sin embargo, no ocurre lo mismo con el confort auditivo, sin duda una de las asignaturas pendientes de la construcción arquitectónica, especialmente en nuestro país, tanto en el aspecto normativo como en el formal.

Son varias las causas de que ello sea así. Unas las compartimos con el resto de los países europeos de misma raíz cultural, como la falta de tradición de su inclusión en la teoría arquitectónica, y otras son particulares, como nuestro tardío y mal planteado desarrollo normativo.

No faltan los que consideran que entre las particulares se encuentra nuestra idiosincrasia que nos lleva a ser más tolerantes con el ruido ajeno que los centroeuropeos ${ }^{6}$. Sin embargo, nadie ha demostrado hasta ahora que el español, en igualdad de coste, prefiera la vivienda en la que se oiga a su vecino, o en la que el ruido de la calle le impida mantener una conversación relajada o incluso dormir. Si lo hace así es porque no tiene otra opción. Las razones evidentemente son de otra índole.

Una evolución positiva debería contar, en consecuencia, con una aproximación de nuestra realidad al nivel ya alcanzado en los países más desarrollados de Europa y, por otra, con la resolución conjunta de los problemas comunes todavía sólo planteados.

El primer paso requiere una reconversión profunda de la NBE.CA.88. El segundo una revisión de algunas de las pautas mentales de los arquitectos.

\section{UNA RECONVERSIÓN INAPLAZABLE}

A pesar de haber sido ya comentados en otras ocasiones ${ }^{7}$ parece aconsejable volver sobre los dos aspectos que distancian considerablemente la NBE.CA de sus homólogas europeas. Por un lado, su formulación descriptiva no exigencial y, por otro, sus niveles reales de exigencia.

En relación al ruido aéreo y mediante la prescripción del valor de $\mathrm{R}$, la norma española en realidad indica cuáles han de ser los materiales constituyentes únicamente de los cerramientos que separan los espacios habitables entre sí o de un exterior ruidoso. Es decir, indica cómo se ha de construir el elemento de separación, pero no indica cuál debe ser el aislamiento real entre los dos espacios, es decir, cuál debe ser el objetivo a cumplir.
La experiencia común y estudios cientificos ya añejos, y recogidos en la propia norma ${ }^{8}$, destacan la gran cantidad de factores que se deben añadir al factor $R$ de la pared de separación, para obtener el valor real del aislamiento: masa relativa de las paredes o forjados contiguos, estanquidad real del conjunto condicionada por diversos factores desde fisuras hasta patios de vecindad, etc.

Consciente de ello la norma francesa, pionera en este campo, definió ya en 1969 los objetivos a cumplir, sin entrar en el cómo se alcanzan que queda a juicio y experiencia del constructor, ayudado por los centros de investigación ${ }^{9}$. La consecución real de los objetivos es verificable sólo con mediciones sonométricas in situ.

Por contra, el control del cumplimiento de la norma española puede reducirse a una cata que nos permita comprobar si la pared en cuestión tiene una determinada masa superficial.

Los factores de transmisión indirecta también coadyuvan al aumento del alejamiento de los niveles de exigencia de los europeos. La NBE prescribe para los elementos de separación una $R$ de $45 \mathrm{~dB}(\mathrm{~A})$. Los estudios citados cifran en 4 ó $5 \mathrm{~dB}(\mathrm{~A})$ la pérdida de aislamiento debida a las transmisiones laterales, supuestos unos cerramientos homogéneos en cuanto a masa, y de hasta 8 ó $9 \mathrm{~dB}(\mathrm{~A})$ si son ligeros. Se puede afirmar, en consecuencia, que el aislamiento real será en el mejor de los casos de $41 \mathrm{~dB}(\mathrm{~A})$, y en bastantes ocasiones inferior a 38 ó $37 \mathrm{~dB}(\mathrm{~A})$. Su comparación con los $51 \mathrm{~dB}(\mathrm{~A})$ reales de la norma francesa o los 54 ó $57 \mathrm{~dB}(\mathrm{~A})$ de la nueva DIN $4109^{10}$, no necesita comentario.

Si analizamos lo referente al ruido de impacto las diferencias se agrandan. La norma española planteada exigencialmente en este caso, prescribe que el ruido producido por la máquina de martillos no debe ser superior a los $80 \mathrm{~dB}(\mathrm{~A})$, frente a los $70 \mathrm{~dB}(\mathrm{~A})$ de la francesa o los $53 \mathrm{~dB}(\mathrm{~A})$ (!) de la nueva DIN.

Sin embargo, la diferencia todavía es mayor si se analiza el procedimiento de aplicación, que vuelve a convertir a la NBE en descriptiva, y las prestaciones que la norma atribuye a los forjados habituales. Ensayos ya añejos y publicados repetidamente ${ }^{11}$ demuestran que los forjados habituales de bovedilla de cerámica o de hormigón por sí solos aislan bastante menos que lo anunciado por la norma. Mientras, según la NBE, un forjado habitual, con un pavimento de terrazo, cumple con los niveles exigidos, $80 \mathrm{~dB}(\mathrm{~A})$, la realidad es que el aislamiento que proporciona no puede impedir que el ruido transmitido sea superior a $90 \mathrm{~dB}(\mathrm{~A})$. La diferencia con el máximo ruido permitido de $70 \mathrm{~dB}(\mathrm{~A})$ de los franceses o de $53 \mathrm{~dB}(\mathrm{~A})$ de los alemanes pone claramente de manifiesto las distancias existentes. 
Y es ahora, considerando la posible aproximación a Europa, cuando se echan de menos estudios que nos permitan vislumbrar por dónde han de ir los cambios tecnológicos de la construcción de nuestros edificios para acercarnos a los niveles indicados. Una vez más hemos de prevenir la importación de soluciones no pensadas para nuestro contexto cultural y climático.

Las soluciones inglesas o francesas basadas en muchos casos en pavimentos blandos pero de alta penetración térmica, no son admisibles en la mayor parte de nuestra geografía, ni compatibles con los forjados habituales.

Las soluciones radicales alemanas basadas en losas flotantes ${ }^{12}$ requerirán una puesta al día de numerosos oficios tradicionales y un replanteo serio de los requerimientos dimensionales y de rigidez de los forjados.

Quizá ya sea el momento de plantearse trabajos de investigación coordinada entre especialistas en estructuras y aislamiento. Quizá es el momento de empezar a pensar en forjados muy ligeros de fuerte canto, que combinados con soleras secas de cartón yeso proporcionen cerramientos horizontales desprovistos de las patologías, tanto sonoras como de flechas excesivas, o en otras soluciones hasta ahora no imaginadas.

En cualquier caso, ya es la hora de dejar de considerar la adecuación acústica como algo a añadir a la solución constructiva. Un nivel de exigencia como el alemán sólo se consigue teniendo presente su consecución desde las primeras líneas del proyecto. Sin duda, un notable cambio para las costumbres habituales de los arquitectos.

\section{RUIDO, CLIMA Y ARQUITECTURA}

Pero más que en los cerramientos interiores, donde mayor dificultad presenta la adecuación sonora de los edificios es en las fachadas. El que en un clima como el nuestro la mayor parte de las viviendas estén enfrentadas a un entorno real o potencialmente ruidoso, implica que el objetivo prioritario es resolver el conflicto del aislamiento con la aireación y la ventilación.

La falta de adecuación general de la NBE.CA.88 y la inexistencia de una norma sobre ventilación comportan que éste sea también un camino prácticamente inexplorado en nuestro país. Nuestras peculiaridades climáticas, con la aireación como parte esencial de los parámetros de confort térmico estival, impiden que la también escasa experiencia europea sea de clara aplicación.

$Y$ es aquí donde la interdisciplinaridad es esencial, pero no sólo de los especialistas en las diferentes ramas físicas de la construcción, sino del creador formal por excelencia, el arquitecto, ya que el problema no tiene solución si no se implica en ella la forma arquitectónica ${ }^{13}$.

Y es en este punto donde nuestra tradición de arquitecto integrador de conocimientos debería suponer una clara ventaja respecto el modelo europeo heredado de Beaux-Arts.

Pero no es así. Como puede comprobarse repetidas veces, las habituales pautas mentales de los arquitectos de aquí, también están basadas en la total prioridad de los valores figurativos y estilísticos frente a la adecuación al uso, con lo que en este aspecto, en la práctica, no diferimos mucho de los arquitectos franceses, por ejemplo. Pero con el grave inconveniente para nosotros de no disponer de ningún tipo de especialista en habitabilidad de la edificación, que complemente sus carencias.

El punto clave a dilucidar es si ello es consecuencia de falta de información o más bien se deriva de los esquemas de valores dominantes en la actual teoría arquitectónica.

La sospecha recaé en lo segundo cuando se comprueba que son bien pocos los casos en que el ambiente ruidoso ha generado alguna respuesta auténticamente arquitectónica, y sí la intervención por adición de los especialistas.

Con tal de avanzar un poco en este sentido, se exponen a continuación dos referencias que pretenden aportar algunos indicios al respecto:

- Con la primera se comprueba que podrían haber existido esquemas teóricos más proclives a la integración, además de que la falta de adecuación acústica no es un problema exclusivo del siglo XX, lo que justificaría su olvido en la tradición teórica.

- Con la segunda que, efectivamente, son las pautas derivadas de la Teoría dominante las que dificultan su integración.

Si ello es así, la resolución del problema no será dar más información al arquitecto, sino transformar sus esquemas de valores teóricos.

\section{LA SENSIBILIDAD DE UN TRATADISTA OLVIDADO}

La teoría arquitectónica podría haber asimilado en mayor medida la adecuación al uso, en este caso acústico, si hubiera basado su desarrollo en el tratado de Arquitectura de Juan Bautista Alberti ${ }^{14}$.

http://informesdelaconstruccion.revistas.csic.es 
Contrariamente a lo impuesto por el tratado dominante en la evolución teórica de la Arquitectura, el de Vitrubio ${ }^{15}$, el tratado de Alberti considera los requerimientos de la construcción y de la adecuación al uso entre los factores principales que determinan la forma arquitectónica.

Dejando para otro lugar ${ }^{16}$ el desarrollo más extendido de la cuestión, y siguiendo el hilo del presente artículo, nos limitaremos a lo relacionado con el ruido. En un delicioso pasaje sin continuidad en ningún tratado posterior, e ignorado habitualmente por sus comentaristas, Alberti plantea sabiamente el problema y su solución.

En los capítulos 11 y 13, del libro $X$, en unos párrafos que no tienen consecuentes hasta el siglo Xx, Alberti deja patente la necesidad de considerar todas las percepciones sensoriales. Las soluciones propuestas confirman su sensibilidad hacia el conocimiento del comportamiento de la materia como parte esencial de la labor del arquitecto.

El capítulo 11 "De algunas cosas menudas que hazen para el uso del fuego", incluye la primera definición en la historia de las condensaciones superficiales y su influencia en la comodidad:

"La pared de pedernal es fría y húmeda, porque con su frialdad cuaja el aire, y le vuelve el sudor, pero la piedra arenisca y ladrillo es más cómoda después que estuviere del todo seca." 17

El capítulo 13 "Del vestir las paredes de los palacios, de el calentar los lugares y del reformar y enmendar los vicios de las mismas paredes", además de continuar con consideraciones sobre la comodidad térmica, incluye la experta y poética referencia al problema del aislamiento acústico. Parafraseando al escritor romano Juvenal ${ }^{18}$ formula la cuestión:

"Se puede presentar el inconveniente del que se duele el poeta en una sátira; que os quite el sueño el pasar las carretas por las tortuosas y es. trechas calles y el alboroto de la muchedumbre atareada." 19

Se ha de recordar que durante esa época en Roma estaba prohibido circular carretas pesadas durante el día $y$, en consecuencia, todo el aprovisionamiento de la ciudad se debía realizar durante la noche.

La solución al problema, por medio de una de las bases fundamentales de los mecanismos de aislamiento, Alberti la expone mediante la carta que Plinio el Joven ${ }^{20}$ dedica a Gallus, describiendo su villa en Laurent, frente al Mediterráneo:

(c) Consejo Superior de Investigaciones Científicas

Licencia Creative Commons 3.0 España (by-nc)
"Anexo a estas estancias está el aposento de la noche y el sueño. A él no llegan las voces de los pajes, ni el murmullo de la mar (...). La razón de ello reside en que la pared de la cámara y el muro del jardín están separados por un pasillo intermedio, y la interposición de este espacio vacío absorbe cualquier sonido." 21

Estas y muchas más ideas de Alberti, que buscan estructurar la teoría de la arquitectura integrando todas las posibles funciones de la materia, no tienen apenas trascendencia en las elaboraciones teóricas de los siglos posteriores, incluido el XX. En éstas, a partir de la ruptura entre la esfera de lo útil y la de lo bello que se instaura al final de la era clásica ${ }^{22}$, domina la concepción perraultiana ${ }^{23}$ que asigna a la materia sólo el requisito de figurar, desproveyéndola de papel en la adecuación al uso.

Por supuesto, no es éste el lugar para seguir intentando demostrar esta afirmación. Pero la siguiente referencia nos permite sospechar que aunque no sea cierta, las cosas parecen ser como si lo fuera.

Servirán de muestra los comentarios de un influyente catedrático de proyectos de Barcelona sobre una de las ya de por sí escasas realizaciones en las que la sensibilidad de los arquitectos genera una respuesta arquitectónica al ambiente ruidoso.

\section{LA INCREDULIDAD DEL CATEDRÁTICO DE PROYECTOS}

En una de sus lecciones, a la que asistí por casualidad ya siendo profesor, se hizo un exhaustivo repaso de los sistemas compositivos de fachada. Entre los definidores del cambio entre la tradición clásica y la modernidad, se destacó la sustitución del papel estático y representativo de la fachada clásica, por una manera de percibirla a través del movimiento del espectador, que comportaba un nuevo carácter de envolvente de la totalidad del volumen del edificio. Uno de los medios para conseguirlo era la creación de texturas con los elementos propios de las fachadas, pero sin que se reflejara en el exterior la interioridad del edificio.

Los ejemplos aducidos se iniciaron con el Like Shore Drive de Mies y alcanzaron a la Lever House. En ningún momento se hizo referencia a razones o contrarrazones derivadas del uso, como protección térmica, solar, ventilación, etc. Todo discurrió por los terrenos de lo figurativo.

Sin embargo, en uno de los casos analizados sí se hizo referencia a la protección auditiva de los ocupan- 
tes del edificio, concretamente al analizar las fachadas del conjunto de viviendas londinense de Robin Hood Gardens, obra del matrimonio Alison y Peter Smithson.

Según quedó razonado en las referencias de las publicaciones del momento ${ }^{24}$, varias disposiciones de la fachada pretenden conseguir que, con un nivel exterior de $75 \mathrm{~dB}(\mathrm{~A})$ y manteniendo las ventanas entreabiertas, el nivel interior no sobrepase los $50 \mathrm{~dB}(\mathrm{~A})$. Desde dinteles recubiertos de materiales absorbentes, hasta unos maineles en saledizo de dos plantas de altura, que además consiguen disminuir las transmisiones entre viviendas por el exterior, ya que las ventanas son contiguas.

Pues bien, el comentario que mereció este esfuerzo integrador entre adecuación acústica y figurativa fue el siguiente:

\begin{abstract}
"Aunque la explicación de los autores es que todos estos elementos colocados desordenada. mente, o más o menos ordenadamente, son para romper el ruido de una autopista cercana, nosotros sabemos que responde a una voluntad de entender la fachada, no como una clara distinción de la ventana de la composición global, sino como una textura, como si fuera un tejido o un empaquetado general." 25
\end{abstract}

Los esquemas teóricos dominantes no sólo tienden a ignorar la adecuación al uso como objetivo prioritario, sino que se mantienen incrédulos frente a aquéllos que contravienen la tendencia.
Los efectos son evidentes. Si algún alumno, siguiendo las indicaciones de los profesores de Cons'rucción o de Acondicionamientos, habia pensado en su próximo proyecto seguir un camino parecido al de los Smithson, en aquel momento cambió de opinión.

Aquellos alumnos hoy serán profesionales con tres o cuatro años de experiencia.

Este ya añejo episodio, que no pretende más que intentar representar una situación general no limitada a la acústica, es bien poca cosa en comparación con otros más actuales. Ahora, en estos tiempos postdogmáticos, algunas tendencias dominantes parecen provenir de profesores que pueden llegar a defender la supremacía de la magia sobre la razón.

\section{Pero eso ya es para otro artículo.}

Una vez más ${ }^{26}$ parece claro que para conseguir, desde la Universidad, un progreso en el mundo de laconstrucción arquitectónica, no es suficiente favorecer investigaciones que nos den resultados de aplicación inmediata o patentes industriales. Es ya imprescindible dar prioridad a estudios que indiquen cómo incidir convincentemente, si es todavía posible, en los criterios, valores y pautas mentales de los que tienen la facultad de decidir la forma arquitectónica.

Quizá sea precisamente la integración europea, con la que hemos iniciado el artículo, la que propicie ese progreso a través de la renovación de los planes de estudios de los profesionales de la edificación.

No desperdiciemos la ocasión.

\section{NOTAS}

1 Es un tema tratado desde hace años en todos los números de la revista C.S.T.B. Magazine (que, junto los Cahier du C.S.T.B., es la publicación francesa equivalente a "Informes de la Construcción”). En el número de mayo del presente año: Pierre Chemilier "Directive Pro. duits de Construction: la mise en oeuvre progresse", y en el reciente de julio-agosto, Stéphane Auzou, "L'acoustique du bâtiment dans l'Europe de demain. Le document interpretatif acoustique".

2 Como muestra de lo afirmado puede aportarse la larga tradición investigadora del C.S.T.B., divulgada mediante numerosísimos articulos desde un lejano "Absortion du son par les structures a panneaux perforés", de J. Brilloouin, de enero de 1949, hasta los más recientes en “C.S.T.B. Magazine”, núm. 42, de marzo de 1991, "Le confort du logement. Vers une compatibilité entre la thermique et l'acoustique", y el conjunto de artículos del núm. 46, de julio-agosto.
También se comprueba que el interés no es sólo de los centros de investigación, sino de los profesionales y de los consumidores. Por ejemplo, véase "Le bruit: probléme majeur des logements français", en “Les Cahiers Techniques du Bâtiment”, de diciembre de 1989. Por contra, es difícil encontrar indicios de interés por el tema en Es. paña durante los veinte últimos años. Algunas de las pocas excepciones se referencian en notas más adelante.

3 Véase al respecto J. Lugez, “La durabilité des materiaux et des ouvrages. Une reflexión dans le cadre de la Directive Produits de la Construction”, en “C.S.T.B. Magazine”, núm. 35, junio 1990.

4 A las dificultades de unificación culturales y climáticas, se añaden las derivadas de los legítimos intereses de los fabricantes de productos de la construcción. Y precisamente uno de los factores que 
pueden llegar a favorecer a unos en detrimento de otros son los sistemas y métodos de medición de las prestaciones. En lo referente a la acústica, véanse los artículos de Jacques Roland, "Acoustique: comment evoluent les regles européens", en Les Cahiers Techniques du Bâtiment, núm. 128, julio-agosto de 1991, e "Indices acoustiques européens. Solutions en vue", en el núm. 46 del C.S.T.B. Magazine. Se delinean claramente dos áreas de interés casi antagónicas: la francesa, a la cual pertenecemos, y la alemana. Véase en relación a esta última afirmación el articulo de Bruno Suner "Les bouches d'air alterent l'acoustique" en Les Cahiers Techniques du Bâtiment, núm. 124, marzo 1991, y el ya citado de Stéphane Auzou.

5 Uno de los escritos que inició la revisión, por parte de la crítica institucionalizada, de las graves carencias ambientales de la arquitectura, surgida del Movimiento Moderno, fue el de Reyner Banham "The architecture of the well-tempered environement", Londres, 1969. Trd. cast. Buenos Aires, 1975. A pesar de considerar en varios pasa. jes la percepción sonora, no le presta atención, y se centra casi ex. clusivamente en la térmica y sus consecuentes instalaciones.

Quizá a la placidez acústica de nuestros edificios urbanos le falta algo asi como su Reyner Banham.

6 Eric Baumlin y otros: "Les tendences techniques dans le secteur de la construction en Europe", Cahiers du C.S.T.B., núm. 2.492, de mayo de 1991. En el apartado dedicado a España, página 30, afirman: 'Au plan de l'acoustique, la mise en place d'une nouvelle reglemen. tation, il y a environ 7 ans, s'est traduit par une prise de conscience; dans ce pays particulièrment bruyant où, sur ce point, les habitants sont par nature tolérants, il ne s'agissait pas au départ d'une exigen. ce fondamental'.

7 A. Casals y J. L. González, “Una norma ambigua. La NBE.CA.82, aprobada...", en CAU, núm. 81, de noviembre de 1982.

J. L. González, “Aislar, siguiendo la normativa española no es suficiente", en Correo de la Construcción, de 13 de septiembre de 1985.

8 En el apartado de la NBE.CA.88, "1.35.3 Influencia de los elementos constructivos adyacentes, transmisiones indirectas", queda todo perfectamente explicado.

9 El cumplimiento de la reglamentación francesa está apoyado por documentos como "Exemples de solutions pouvant satisfaire au re. glement de la construction (acoustique) et (ou) aux definitions du la. bel confort acoustique", Cahiers du C.S.T.B., núm. 1.373, abril 1976, y por numerosísimas publicaciones de divulgación posteriores.

Según un control del cumplimiento normativo en la construcción real, la tasa de cumplimiento ha pasado de un $7 \%$ en 1973 a más de un $70 \%$ en 1987. Ver Jean-Pierre Bardy, "Une prise en compte par les pouvoirs publics", en C.S.T.B. Magazine, núm. 46. Según este autor, una razón del éxito es el carácter exigencial de la norma, que permite a cada constructor aplicar su propia tecnología.

10 La puesta en marcha de la nueva versión de la norma DIN 4109, todavia más exigente, ha dejado irritados a los especialistas france. ses: Robert Josse, “Lutte contre le bruit: I'Allemagne prend l'avance", en C.S.T.B. Magazine, núm. 32, marzo 1990.

El aumento de los niveles en Francia es cuestión de poco tiempo. En el número 46 de esta revista, ya citado, se da cuenta de una ope. ración piloto en la que se ha experimentado el cumplimiento de nue. vos niveles de exigencia con un sobrecosto de construcción de sólo el $1 \%$, insistiéndose en la insuficiencia de la normativa actual.

11 S. Auzou, "Etude des caractéristiques acoustiques de materiaux et d'equipements", Cahiers de C.S.T.B., núm. 1.397, octubre de 1976 , pág. 61. G. Pinçon, “Amelioration acoustique des logements", CATED, París, 1988.

Un forjado de bovedilla cerámica y masa superficial $320 \mathrm{~kg} / \mathrm{m}^{2}$ da un Ln de $95 \mathrm{~dB}(\mathrm{~A})$, contra los $86 \mathrm{~dB}(\mathrm{~A})$ atribuidos por la NBE.
El error proviene de aplicar a los forjados de bovedillas la expresión $R+L n=135$ dB que sólo es válida para las losas macizas de hormigón.

12 Las diferentes formas de medición del ruido de impacto también pueden dar preferencia a determinados sistemas constructivos. EI alemán favorece a las losas flotantes. Los fabricantes franceses de suelos ligeros luchan por que no aumente la exigencia y que se man. tenga el método de medida francés. Véanse los artículos ya citados de Jacques Roland.

13 Véase F. Ramón, "Control de ruidos en una situación urbana", Madrid, 1976.

A. Casals y J. L. González, “Manual de Acústica y Edificación”, revista $C A U$, núms. 72, 73, 74, 75 y 76, de 1981.

14 J. B. Alberti, “Re Edificatoria”, Firenze, 1485. Traducción realizada por Lozano en 1582. Los párrafos que se han extractado han sido reelaborados para hacer inteligible el castellano de Lozano. Las referencias son de la edición de Madrid de 1582 (o en sus versiones facsímiles, Oviedo 1975, Valencia 1977).

15 La influencia, a través de su famosa tríada, de su restrictiva ma. nera de integrar la construcción en la arquitectura, llega a destaca. dos teóricos contemporáneos como Christian Norberg-Schulz, en sus "Intencions in Architecture", Oslo, 1963, o Ludovico Quaroni, en su “Progettare un edificio. Otto lezioni di architettura”, Milano, 1977, u otros no tan destacados pero más recientes como Giovanni Morabito, en su “Forme e tecniche dell'architettura moderna", Roma, 1990.

16 Este es uno de los temas dominantes en la tesis doctoral del autor del artículo.

J. L. González, "Un estudio sobre la desvinculación de los contenidos de construcción de los tratados de arquitectura del siglo XVI al XIX”, UPC, Barcelona, 1987. Se prevé su publicación durante el próximo año.

17 Alberti, op. cit., pág. 336.

18 Juvenal, Sátiras, Sátira III.

19 Alberti, op. cit., pág. 338.

20 Plinio el Joven, Cartas, Libro II, Carta 17.

21 Alberti, op. cit., pág. 338.

22 A. Picon, "Histoire de l'architecture, histoire des sciences et des techniques", en "Cahiers de la recherche architectural”, núm. 26, 2. trimestre de 1990.

Antoine Picón, ingeniero de caminos y arquitecto francés, ha desarrollado interesantes investigaciones sobre la relación entre arquitectura y construcción.

23 Fué la versión abreviada del tratado de Vitruvio escrita por el na. turalista Claude Perrault, la que estableció la definitiva y restrictiva versión de la tríada.

Ver tesis doctoral de J. L. González.

24 Architectural Design, Septiembre de 1972, pp. 557 a 573. También se hace referencia aunque sólo desde la esfera figurativa en Peter Einsenman, "From Golden Lane to Robin Hood Gardens;...” en Oppositions n: 1 de septiembre de 1973.

25 Apuntes recogidos por el autor en la clase impartida en catalán el 12 de enero de 1982 en la ETSAB.

26 J. L. González, "Evolución normativa y progreso en construcción arquitectónica", en Informes de la Construcción 411 ene-feb. 1991. 